\title{
Overtaking collisions of double layers and solitons: Tripolar structures and dynamical polarity switches
}

Cite as: Phys. Plasmas 27, 062303 (2020); doi: 10.1063/5.0003493

Submitted: 2 February 2020 - Accepted: 22 May 2020 .

Published Online: 12 June 2020

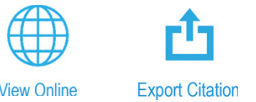

C. P. Olivier ${ }^{1, a)}$ (D) and F. Verheest ${ }^{2,3}$ iD

\begin{abstract}
AFFILIATIONS
'Department of Mathematics and Applied Mathematics, North-West University, Mafikeng Campus, Mmabatho 2745, South Africa

${ }^{2}$ Sterrenkundig Observatorium, Universiteit Gent, Krijgslaan 281, B-9000 Gent, Belgium

${ }^{3}$ School of Chemistry and Physics, University of KwaZulu-Natal, Scottsville, Pietermaritzburg 3209, South Africa
\end{abstract}

a) Author to whom correspondence should be addressed: carel.olivier@nwu.ac.za

\begin{abstract}
Overtaking collisions between double layers and solitons are investigated through the numerical simulation of the Gardner equation that governs small-amplitude double layers. The results show that the double layer emerges unaffected after the completion of the collision. However, the soliton that emerges from the collision has the opposite polarity of the soliton prior to the collision. In addition, it is shown that a transient tripolar electric field structure forms during the collision. Possible applications to spacecraft observation of electrostatic solitary waves are briefly discussed.
\end{abstract}

Published under license by AIP Publishing. https://doi.org/10.1063/5.0003493

\section{INTRODUCTION}

Electrostatic solitary waves (ESWs) have been reported in many regions of the Earth's magnetosphere..$^{1-7}$ Here, the term ESW is used to describe spatially localized electric field signatures, as opposed to oscillating or noisy signals. As such, it describes a large range of electric field structures, including unipolar, bipolar, and tripolar structures. In addition, more complicated structures have also been observed, consisting of multiple "wiggles" in the electric field. The most commonly observed ESWs are bipolar electric field structures. In addition, tripolar electric field structures are frequently observed in boundary regions of the magnetoshpere, ${ }^{7-9}$ as well as regions of magnetic reconnection. ${ }^{10}$ On the other hand, only a small number of reports of unipolar electric field structures have been reported. ${ }^{1}$ These observations are usually interpreted as Bernstein-Greene-Kruskal (BGK) modes that arise from kinetic models with trapped ion and/or electron species. ${ }^{11-13}$

An alternative approach to study such structures is rooted in fluid theory. ${ }^{14}$ Indeed, soliton solutions of fluid theory ${ }^{15,16}$ can account for the observation of bipolar electric field structures. In addition, the double layer solutions of fluid theory ${ }^{17}$ can be used to model the observation of unipolar electric field structures, while supersoliton solutions ${ }^{18-20}$ may explain more complicated electric field structures (it should be noted that, unlike fluid theory, observational papers use the term weak double layer for any structure that results in a shift in the electrostatic potential, including tripolar electric field structures). However, no satisfactory theoretical description for tripolar electric field structures has been established within fluid theory.

In addition, solitary wave collisions are important in the interpretation of electric field observations. This is due to the fact that solitary waves are usually observed in clusters on a distorted background. ${ }^{2,3,5,6,8,9}$ Fluid theory suggests that, unless solitons have identical amplitudes, they propagate at different velocities. As such, a number of theoretical and numerical studies are dedicated to soliton collisions, both for head-on ${ }^{21-27}$ and overtaking collisions. ${ }^{27-30}$ Collisions between solitons and supersolitons were also studied numerically. ${ }^{31,32}$ These studies suggest that some observations of more complicated electric field structures may be the result of soliton collisions.

Interestingly, collision properties of double layers received less attention. To address this, we study the characteristics of collisions of double layer solutions overtaking solitons. Our study is performed in the small-amplitude regime via reductive perturbation analysis. Since reductive perturbation analysis is limited to nonlinear structures that propagate slightly faster than the acoustic speed, the study of head-on collisions is beyond the scope of this approach.

Two important results were obtained. First, after the collision, a soliton of opposite polarity emerges. Second, tripolar electric field 
transients form during the collision. These findings have interesting consequences that is discussed in more detail in the latter part of the paper.

This paper is structured as follows: in Sec. II, we review the main characteristics of the Gardner equation that supports small-amplitude double layer solutions. Section III is devoted to the setup of the numerical scheme in order to simulate the collision. In Sec. IV, the results of the simulations are presented. The implications of these results are discussed in more depth in Sec. V. In Sec. VI, the main conclusions from the paper are summarized, and extensions to the study are proposed.

\section{SMALL-AMPLITUDE SOLITON AND DOUBLE LAYER SOLUTIONS}

The Gardner equation has been derived in the description of many plasma models by means of the reductive perturbation method. ${ }^{33-35}$ Following the introduction of appropriate coordinate stretching variables, one obtains a Gardner equation of the following form:

$$
\phi_{1 \tau}+\frac{1}{2} \phi_{1 \xi \xi \xi}+a \phi_{1} \phi_{1 \xi}+b \phi_{1}^{2} \phi_{1 \xi}=0
$$

where the electrostatic potential $\phi$ is expanded as

$$
\phi=\varepsilon \phi_{1}+\varepsilon^{2} \phi_{2}+\cdots,
$$

$\xi=\varepsilon\left(x-M_{0} t\right)$ is a moving frame, $M_{0}$ is an acoustic speed, and $\tau=\varepsilon^{3} t$ is a slow time scale.

When $b<0$ (poor near-critical composition), ${ }^{36}$ the Gardner equation has soliton solutions of the following form:

$$
\phi_{1 s}(\xi, \tau)=\frac{\delta M}{\frac{a}{6}+\sqrt{\frac{a^{2}}{36}+\frac{b \delta M}{6}} \cosh [\sqrt{2 \delta M}(\xi-\delta M \tau)]},
$$

where

$$
0<\delta M<\delta M_{D L}
$$

with

$$
\delta M_{D L}=\frac{a^{2}}{6|b|}
$$

Here, $\delta M$ represents the amount by which the soliton velocity exceeds the acoustic speed $M_{0}$. That is, if $M$ is the Mach number (as defined in Sagdeev analysis), the velocity $\delta M$ satisfies the relation

$$
\varepsilon^{2} \delta M=M-M_{0} .
$$

As such, we refer to $\delta M$ as the excess velocity. While $\varepsilon$ may be treated as a bookkeeping parameter, its presence in the relation (6) reminds one that the excess velocity must be sufficiently small in order to satisfy the small-amplitude condition.

At the critical excess velocity $\delta M=\delta M_{D L}$, the Gardner equation has double layer solutions,

$$
\phi_{1}(\xi, \tau)=-\frac{a}{2 b}\left[1 \pm \tanh \sqrt{-\frac{a^{2}}{12 b}}\left(\xi-\frac{a^{2}}{6|b|} \tau\right)\right] .
$$

Following Torvén, ${ }^{37}$ we choose the negative - sign, so that the boundary condition $\phi \rightarrow 0$ when $\xi \rightarrow+\infty$ is satisfied. We refer to this solution as $\phi_{1 d}$, so that

$$
\phi_{1 d}(\xi, \tau)=-\frac{a}{2 b}\left[1-\tanh \sqrt{-\frac{a^{2}}{12 b}}\left(\xi-\frac{a^{2}}{6|b|} \tau\right)\right] .
$$

An important point regarding the boundary conditions of these solutions must be noted. While soliton solutions (3) satisfy the following set of boundary conditions:

$$
\phi \rightarrow 0, \text { when } \xi \rightarrow \pm \infty,
$$

the double layer solution does not satisfy these conditions. This is due to its asymptotic behavior, where $\phi_{1 d} \rightarrow-\frac{a}{b}$ when $\xi \rightarrow-\infty$. It follows directly that the set of boundary conditions that supports a double layer solutions is given by

$$
\phi \rightarrow-\frac{a}{b}, \text { when } \xi \rightarrow-\infty ; \phi \rightarrow 0, \text { when } \xi \rightarrow+\infty .
$$

This consideration is crucial for the design of an appropriate numerical scheme.

It should also be noted that the existing criterion (4) shows that double layer solutions propagate at the upper limiting velocity of solitons. This means that the double layers cannot be overtaken by solitons. Instead, overtaking collisions can only occur when double layers overtake solitons. This reflects the characteristic of the Gardner equation that it does not support supersoliton solutions. In the presence of supersolitons, there exist solitons and supersolitons that propagate faster than the double layer solution, so that the double layer may be overtaken by other nonlinear structures. To study such collisions, one would have to resort to the modified Gardner equation. ${ }^{31}$ However, such collisions are beyond the scope of the present paper.

\section{NUMERICAL SCHEME}

To simulate the collision of solitons and double layers, one has to obtain an initial condition that can generate this situation. Once the initial condition is chosen, one must develop a computer code to integrate the equation numerically. For the simulations, we consider the normalized form of the Gardner equation (1) with $a=1$ and $b=-1$, given by

$$
\phi_{1 \tau}+\frac{1}{2} \phi_{1 \xi \xi \xi}+\phi_{1} \phi_{1 \xi}-\phi_{1}^{2} \phi_{1 \xi}=0
$$

In order to produce an initial condition that will generate soliton solutions, we use (3) with $\tau=0$. The resulting initial conditions are then given by

$$
g_{s}(\xi)=\phi_{1 s}(\xi, 0)=\frac{6 \delta \mathrm{M}}{1+\sqrt{1-6 \delta \mathrm{M}} \cosh (\sqrt{2 \delta \mathrm{M}} \xi)} .
$$

Similarly, we use the solution of the double layer (8) with $\tau=0$ to obtain an initial condition $g_{d}(\xi)$ that will produce a double layer solution, namely,

$$
g_{d}(\xi)=\frac{1}{2}\left(1-\tanh \sqrt{\frac{1}{12}} \xi\right)
$$


Both the soliton and double layer initial conditions are centered at $\xi=0$. In order to simulate a collision, we combine these two initial conditions by shifting the soliton initial condition so that it is centered far away from the center of the kink. In our simulations, we typically shifted the soliton 100 units of $\xi$ to the right of the double layer's center. The resulting initial condition is then given by

$$
g(\xi)=g_{d}(\xi)+g_{s}(\xi-100) .
$$

As an example, we show the initial condition obtained by the formula (14) for a soliton with excess velocity $\delta M=0.9 \delta M_{D L}$. The resulting initial condition is shown in Fig. 1. Here, we see that the (faster) double layer is situated to the left of the (slower) soliton solution. Since both structures propagate from left to right, one can expect an overtaking collision during the evolution of the system. Also note that the soliton is initially situated in a nearly flat background. The result is that the soliton initially propagates similarly to a soliton in the absence of the double layer. The soliton only deforms significantly when the double layer overtakes it.

Once the initial condition is chosen, we can solve the Gardner equation (11) numerically. The first step is to truncate the spatial interval. This is done by choosing a large but finite interval length $L$, and solving the equation on the interval $\xi \in\left[-\frac{L}{2}, \frac{L}{2}\right]$. To deal with the boundary conditions, we apply a truncated analog of the boundary conditions of the double layer solution (10), resulting in the following set of boundary conditions:

$$
\phi\left(-\frac{L}{2}, \tau\right)=1, \quad \frac{\partial \phi}{\partial \xi}\left(-\frac{L}{2}, \tau\right)=0,
$$

and

$$
\phi\left(\frac{L}{2}, \tau\right)=0, \quad \frac{\partial \phi}{\partial \xi}\left(\frac{L}{2}, \tau\right)=0,
$$

for all $\tau \geq 0$. After truncation, we introduce a grid on the spatial domain. We used standard second order centered difference formulas to approximate the $\xi$-derivative terms, and a fourth-order Runge-Kutta scheme to integrate with respect to $\tau$. For most of the simulations, we used a $\xi$ step size of $\Delta \xi=0.1$. For this choice, $\Delta \tau=$

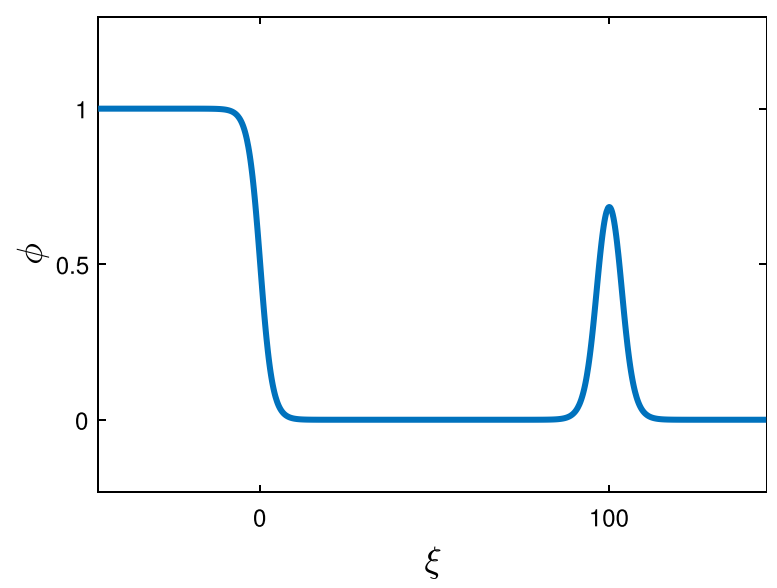

FIG. 1. Initial condition (14) for the simulation. Here, the excess velocity of the soliton is $\delta M=0.9 \delta M_{D L}$
0.001 was sufficiently small to ensure the stability of the numerical scheme. In addition, the $\tau$-resolution is sufficiently high to ensure that the Courant-Friedrichs-Lewy condition ${ }^{38}$ is observed, namely that $U_{\max } \Delta \xi / \Delta \tau<1$, where the maximum wave propagation speed is the double layer velocity, given by $U_{\max }=1 / 6$.

In general, larger soliton velocities require a longer $\tau$ - integration in order for the collision to occur. This, in turn, requires a longer $L$-interval. As such, we used different interval lengths, depending on the excess velocity of the soliton. Finally, in order to utilize the full $\xi$ range, we shifted the initial condition left. We typically used the initial condition

$$
\phi(\xi, 0)=g\left(\xi+\frac{L}{2}-50\right),
$$

for $g(\xi)$ defined in (14).

Our numerical code was validated by means of the exact double layer solution (7). To do this, we simulated the solution by using the initial condition (13). Strong agreement between the numerical and analytical solutions was observed.

\section{RESULTS}

We performed simulations for many different soliton excess velocities. The behavior of the collisions agrees qualitatively for all the choices of excess velocity that were considered. As such, we provide a detailed example for one choice of excess velocity of the soliton, namely $\delta M=0.95 \delta M_{D L}$. Here, we used an interval length of $L=3000$.

In Fig. 2, we show the solution for six different values of $\tau$. Figure 2(a) shows the initial condition at $\tau=0$. Here, we see that the soliton is initially situated 100 units of $\xi$ in front of the double layer. In Fig. 2(b), the solution is shown at $\tau=7000$. Clearly, the double layer is catching up with the soliton, so that the distance between the two structures is less than 50 units of $\xi$. In Fig. 2(c), the solution is shown at $\tau=10190$. At this stage, there is no longer a gap between the soliton and the double layer. The two background layers are divided by a bipolar structure, consisting of two local extremes, with a local minimum on the left, and a local maximum situated on the right.

During the collision, both these local extremes increase with $\tau$. When $\tau=10620$, shown in Fig. 2(d), the distance between the local maximum and the bottom layer is the same as the distance between the local minimum and the top layer. As $\tau$ is increased further, the local extremes increase further, as is clear from Fig. 2(e), showing the solution at $\tau=11050$. This process continues until the local maximum approaches the top layer at $\phi=1$. Thereafter, the soliton separates from the structure, while the original double layer re-emerges. Importantly, the soliton has an opposite polarity, in other words, it is a dip instead of an enhancement in $\phi$, as is seen from Fig. 2(f), showing the solution at $\tau=16000$. As $\tau$ increases further, the distance between the altered soliton and the double layer increases. After the collision, the soliton and double layer propagate with the same speed as before the collision.

The electrostatic potential $\phi$ can be used to construct the electric field $E$ from the following relation:

$$
E=-\nabla \phi=-\frac{\partial \phi}{\partial x} .
$$

Since $\frac{\partial}{\partial x}=\varepsilon \frac{\partial}{\partial \xi}$ and $\phi=\varepsilon \phi_{1}+\varepsilon^{2} \phi_{2}+\cdots$, it follows that the electric field can be expanded as 

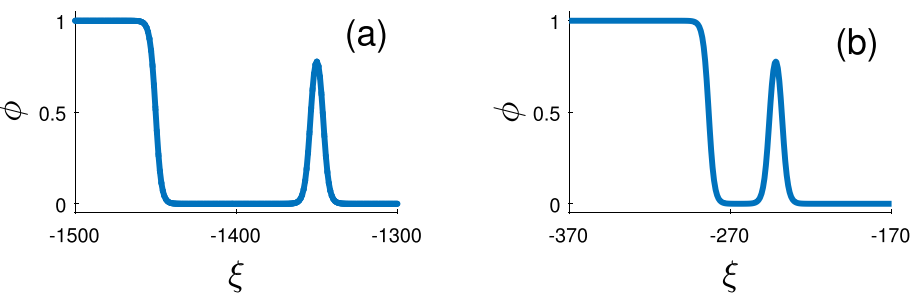

(d)

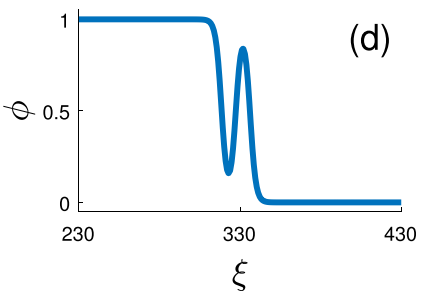

(a)

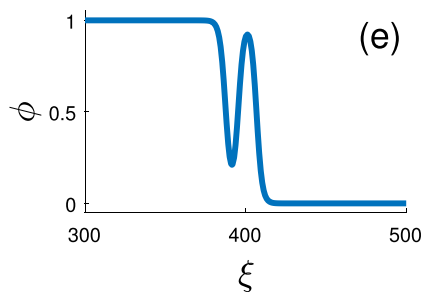

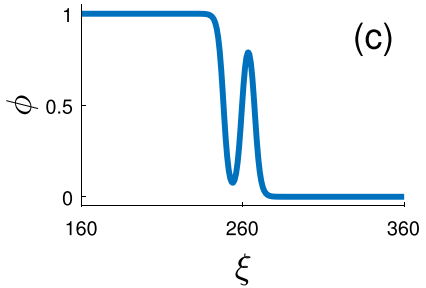

(c)

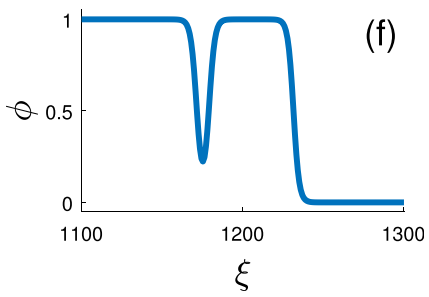

FIG. 2. Snapshots of the electrostatic potential profiles during the collision of the double layer and soliton with excess velocity $\delta M=0.95 \delta M_{D L}$ at the following choices of $\tau$ : (a) $\tau=0$, (b) $\tau=7000$, (c) $\tau=10190$, (d) $\tau=10620$, (e) $\tau=11050$, and (f) $\tau=16000$.

$$
E=\varepsilon^{2} E_{1}+\varepsilon^{3} E_{2}+\cdots
$$

where

$$
E_{j}=-\frac{\partial \phi_{j}}{\partial \xi},
$$

for $j=1,2, \ldots$.

Using Eq. (20) for $j=1$, we numerically differentiated the solutions shown in Fig. 2 to produce the electric field structure during the collision. The results reveal the same progression as Fig. 2, namely the double layer overtaking the soliton, the collision, and the reemergence of the soliton and double layer. However, these results reveal the formation of a tripolar electric field structure that forms during the collision, as is seen in panels (c)-(e). In panels (c) and (e), we see asymmetric tripolar electric field structures, whereas panel (d) shows an almost symmetric tripolar structure. It is worth mentioning that, despite these asymmetries, panels (c)-(e) look very similar, demonstrating that the transient tripolar electric field structure changes slowly during the course of the collision process.

\section{DISCUSSION}

The results shown for the electrostatic potential have interesting and important consequences. In addition, the electric field profiles have different but equally important consequences. We therefore consider each set of results separately.

\section{A. Electrostatic potential: Dynamical polarity switching}

The results shown in Fig. 2 highlight the fact that the polarity of the soliton switches during the collision. Before the collision, the soliton has a positive polarity, i.e., the soliton results in an increase in the electrostatic potential. However, the soliton that forms after the collision has a negative polarity, i.e., the soliton results in a decrease in the electrostatic potential.
To analyze this change in polarity, we note that the soliton propagates on a different background after the collision than before it. Before the collision, the soliton propagated on the background $\phi \rightarrow 0$ when $\xi \rightarrow+\infty$. However, after the collision, the background is provided by the other boundary condition, namely $\phi_{1} \rightarrow \phi_{D L}$ when $\xi \rightarrow-\infty$, where $\phi_{D L}=1$ for the normalized coefficients.

Based on this, we introduce a new variable,

$$
\psi=\phi_{1}-\phi_{D L}
$$

This variable corresponds to the difference between the electrostatic potential and the associated background layer $\phi_{D L}$. Since $\phi_{D L}=1$, we substitute $\phi_{1}=1+\psi$ into the normalized Gardner equation (11). After evaluating the derivatives, one obtains

$$
\psi_{\tau}+\frac{1}{2} \psi_{\xi \xi \xi}+(1+\psi) \psi_{\xi}-\left(1+2 \psi+\psi^{2}\right) \psi_{\xi}=0 .
$$

Following a basic algebraic manipulation, one obtains the following evolution equation for $\psi$ :

$$
\psi_{\tau}+\frac{1}{2} \psi_{\xi \xi \xi}-\psi \psi_{\xi}-\psi^{2} \psi_{\xi}=0 .
$$

This equation describes solitons that propagate in the wake of the double layer in the limit where the distance between the soliton and the double layer is sufficiently large.

A comparison between (11) and (23) shows that the only difference between the two equations is a change in the sign of the quadratic nonlinear term. From a symmetry between the two equations, it follows that $\psi=-\phi_{s}$ is a solution of (23), for $\phi_{s}$ given by (3).

The results imply that the change of polarity can simply be attributed to the change in the background electrostatic potential. Before the collision, the background to the right of the double layer is approximately the equilibrium condition $\phi=0$, so that the soliton is governed by the Gardner equation (11). After the collision, the soliton propagates on the background on the left of the boundary condition, namely $\phi_{1}=\phi_{D L}$. As a result, the soliton is (approximately) governed 
by the Gardner equation (23), resulting in a soliton with opposite polarity.

It is worth noting that the Gardner equation (11) supports no negative polarity solitons. It is therefore interesting that negative polarity solitons may still exist in the wake of a double layer. This presents an alternative mechanism to explain, for example, the observation of negative polarity ion-acoustic solitons in space plasmas. ${ }^{1,6,39}$

\section{B. Electric field potential: Tripolar transients}

There are many spacecraft observations of tripolar electric field structures. In more active regions of the Earth's magnetosphere, it was shown that the number of tripolar structures are around one tenth of that of bipolar structures. ${ }^{8,10}$ In the following, we explore the possibility that the tripolar electric field transient structures from the simulations are related to these observations.

To start off, we consider the results from Li et al. ${ }^{10}$ They paid special attention to the waveforms of the tripolar electric field structures. It was shown that the majority of the observed structures are asymmetric. The results from our simulations also form asymmetries, as is clear from Figs. 3(c) and 3(e). It is only halfway through the collision that a symmetric waveform is recovered, as shown in Fig. 3(d). These results agree well with the above-mentioned observations.

The question then arises: how long can these transient structures retain their shape? To investigate this, we considered the lifetime of these structures for different soliton velocities. Our analysis shows that the lifetime of the transients increases with the velocity of the soliton. This comes as no surprise, as one would expect that the double layers would take longer to overtake a faster soliton.

In order to measure the duration of the tripolar transients, we define the transient lifetime as $L T=\tau_{\text {end }}-\tau_{\text {start }}$. Here, $\tau_{\text {start }}$ is defined as the critical value of $\tau$, where the minimum value between the soliton peak and the double layer is 0.01 . Similarly, $\tau_{\text {end }}$ is defined as the critical value of $\tau$, where the maximum value between the trough of the (newly formed) soliton and the double layer is 0.99 .
The two critical values of $\tau$ are illustrated in Figs. 4(a) and 4(b). Here, the results are shown for the collision of a double layer and a soliton with excess velocity $\delta M=0.9 \delta M_{D L}$. Figure 4(a) shows the solution at $\tau=4675$. In the main figure, the minimum of the potential between the soliton peak and the double layer is indicated with a small rectangle. The subplot (indicated with the arrow) shows the region of this minimum on a smaller scale, confirming that $\tau_{\text {start }}=4675$ for this simulation. Similarly, the solution at $\tau=6165$ is shown in Fig. 4(b), confirming that $\tau_{\text {end }}=6165$. By subtracting these two critical times, it follows that the lifetime of the tripolar structure for this simulation is given by $L T=1490$ units of $\tau$.

In Fig. 4(c), we show the collision times for different excess soliton velocities. We see a clear increase when $\delta M \rightarrow \delta M_{D L}$. It is interesting to note that the rate of increase is faster than exponential. This suggests that the duration of the tripolar structures is unbounded, with a vertical asymptote at $\delta M=\delta M_{D L}$, as indicated by the dashed line.

It should be mentioned that the computational time increases drastically with increasing $\delta M$. As such, for excess soliton velocities $\delta M>0.95 \delta M_{D L}$, we reduced the resolution to $\Delta \xi=0.5$ and $\Delta \tau=0.01$. For these choices, the results were consistent with the higher resolutions used for smaller excess velocities.

We must caution the reader, however, of the limitations of this study. The small-amplitude and near-critical limitations severely restricts the scope of the study. As such, one would require the simulation of the full fluid model in order to establish these characteristics in the large-amplitude regime, where much more realistic comparisons can be made to satellite observations.

Another important aspect that must be established is the generation of these structures. The method used to simulate these structures, the overtaking collision of double layers and solitons, does not seem viable, as the overtaking may take very long. In addition, spacecraft observations of solitons usually consist of only a single polarity, ruling out the observation of solitons on different sides of a double layer. An alternative possibility is that some localized disturbance immediately gives rise to tripolar transient structures. For this to be valid, one must
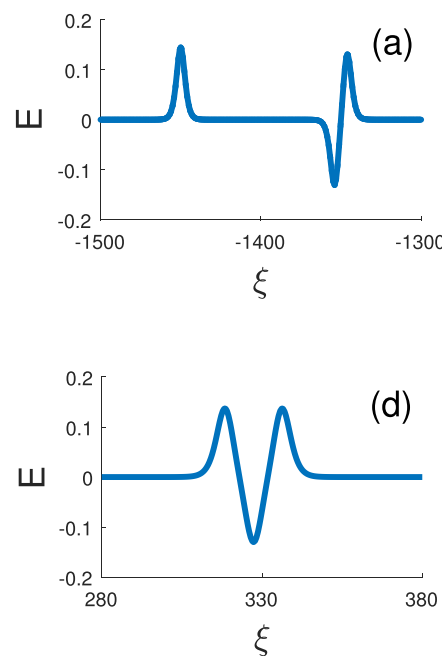
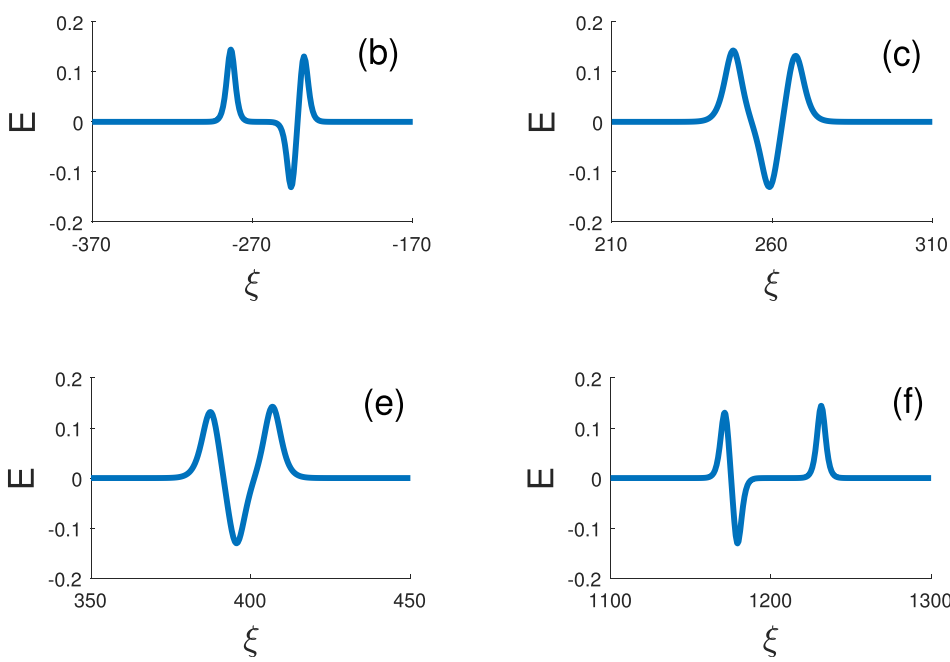

FIG. 3. Snapshots of the electric field $E_{1}$ during the collision of a double layer and soliton with excess velocity $\delta M=0.95 \delta M_{D L}$. The values of $\tau$ for different panels are the same as given in Fig. 2. 

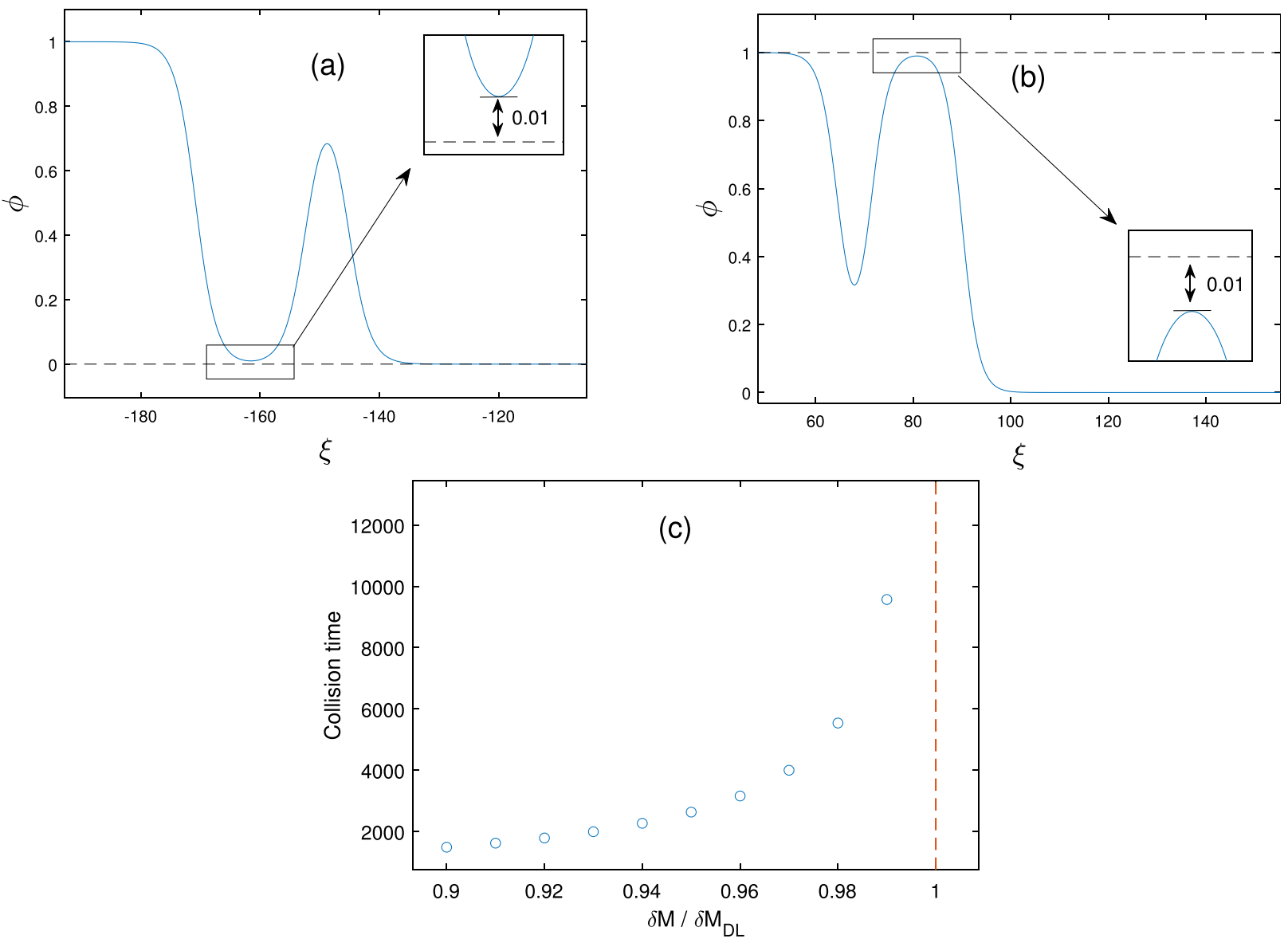

FIG. 4. Examples of the starting time $\tau_{\text {start }}$ and end time $\tau_{\text {end }}$ of the tripolar electric field transient are shown in (a) and (b), respectively. In panel (c), the lifetime of the tripolar electric field transients is shown as a function of the excess velocity of the soliton.

gain more insight into the generation mechanisms of such structures, either from fluid simulations or laboratory experiments.

We would like to point out that the study of these limitations is beyond the scope of this paper. Nevertheless, we argue that these results present a promising possibility to provide a theoretical explanation for the observation of tripolar electric field structures.

\section{CONCLUSIONS AND FUTURE WORK}

In this paper, we performed a simulation study of overtaking collisions between solitons and double layers. The study is limited to the small-amplitude regime where solitons and double layers are governed by the Gardner equation. The two main results are briefly summarized below.

First, we showed that, after the collision, the double layer reemerges without a change in shape or velocity. However, after the collision, a new soliton emerges with an opposite polarity of the soliton that existed prior to the collision. In other words, the collision results in a dynamical polarity switch. This means that solitons in the wake of a double layer have the opposite polarity of those in front of the double layer. This may account for the observation of negative polarity ion- acoustic solitons and positive polarity electron-acoustic solitons in space plasmas.

Second, we showed that tripolar electric field structures form during the collision of the double layer with the soliton. To the best of our knowledge, this is the first time that tripolar electric field structures are reported in fluid theory. Unlike solitons and double layers, these transient structures have a finite lifespan, and will eventually decouple to form a soliton and a double layer. However, our results suggest that the lifetime of the transients are unbounded in the limit when the soliton velocity approaches the double layer velocity. As such, the results predict that these structures can propagate over long distances while retaining a slowly changing tripolar waveform.

The existence of tripolar electric field transients in fluid models are encouraging. However, this is only the first step toward understanding these structures due to the small-amplitude limitation that neglects higher order nonlinear effects on large-amplitude tripolar electric field transients. These effects can be studied by means of fluid simulations. If qualitative agreement can be established, it would be very interesting to determine if a correlation exists between the results obtained from magnetospheric fluid models and observations. 


\section{DATA AVAILABILITY}

Data sharing is not applicable to this article as no new data were created or analyzed in this study.

\section{REFERENCES}

${ }^{\mathbf{T}}$ M. Temerin, K. Cerny, W. Lotko, and F. S. Mozer, Phys. Rev. Lett. 48, 1175 (1982).

2J. R. Franz, P. M. Kintner, and J. S. Pickett, Geophys. Res. Lett. 25, 1277, https://doi.org/10.1029/98GL50870 (1998).

${ }^{3}$ R. E. Ergun, C. W. Carlson, J. P. McFadden, F. S. Mozer, G. T. Delory, W. Peria, C. C. Chaston, M. Temerin, I. Roth, L. Muschietti, R. Elphic, R. Strangeway, R. Pfaff, C. A. Cattell, D. Klumpar, E. Shelley, W. Peterson, E. Moebius, and L. Kistler, Geophys. Res. Lett. 25, 2041, https://doi.org/10.1029/ 98GL00636 (1998).

${ }^{4}$ S. D. Bale, P. J. Kellogg, D. E. Larson, R. P. Lin, K. Goetz, and R. P. Lepping, Geophys. Res. Lett. 25, 2929, https://doi.org/10.1029/98GL02111 (1998).

${ }^{5}$ H. Kojima, Y. Omura, H. Matsumoto, K. Miyaguti, and T. Mukai, Nonlinear Process. Geophys. 6, 179 (1999).

${ }^{6}$ S. R. Bounds, R. F. Pfaff, S. F. Knowlton, F. S. Mozer, M. A. Temerin, and C. A. Kletzing, J. Geophys. Res. 104, 28709, https://doi.org/10.1029/1999JA900284 (1999).

7J. S. Pickett, L.-J. Chen, S. W. Kahler, O. Santolík, D. A. Gurnett, B. T. Tsurutani, and A. Balogh, Ann. Geophys. 22, 2515 (2004).

${ }^{8}$ J. S. Pickett, S. W. Kahler, L.-J. Chen, R. L. Huff, O. Santolík, Y. Khotyaintsev, P. M. E. Décréau, D. Winningham, R. Frahm, M. L. Goldstein, G. S. Lakhina, B. T. Tsurutani, B. Lavraud, D. A. Gurnett, M. André, A. Fazakerley, A. Balogh, and H. Rème, Nonlinear Process. Geophys. 11, 183 (2004).

${ }^{9}$ Y. Hobara, S. N. Walker, M. Balikhin, O. A. Pokhotelov, M. Gedalin, V. Krasnoselskikh, M. Hayakawa, M. André, M. Dunlop, H. Rème, and A. Fazakerley, J. Geophys. Res. 113, A05211, https://doi.org/10.1029/ 2007JA012789 (2008).

${ }^{10}$ S. Y. Li, S. F. Zhang, H. Cai, X. Q. Chen, and X. H. Deng, J. Geophys. Res. 118, 2911, https://doi.org/10.1002/jgra.50209 (2013).

${ }^{11}$ H. Schamel, Phys. Scr. T2 1, 228 (1982).

${ }^{12}$ D. Jovanović, P. K. Shukla, and G. Morfill, J. Plasma Phys. 71, 203 (2005).
${ }^{13}$ B. Eliasson and P. K. Shukla, Phys. Rep. 422, 225 (2006).

${ }^{14}$ G. S. Lakhina, S. V. Singh, R. Rubia, and T. Sreeraj, Phys. Plasmas 25, 080501 (2018).

${ }^{15} \mathrm{H}$. Washimi and T. Taniuti, Phys. Rev. Lett. 17, 996 (1966).

${ }^{16}$ R. V. Sagdeev, in Reviews of Plasma Physics edited by M. A. Leontovich (Consultants Bureau, 1966), Vol. 4, pp. 23-91.

${ }^{17}$ R. Bharuthram and P. K. Shukla, Phys. Fluids 29, 3214 (1986).

${ }^{18}$ A. E. Dubinov and D. Yu. Kolotkov, Plasma Phys. Rep. 38, 909 (2012).

${ }^{19}$ A. E. Dubinov and D. Yu. Kolotkov, High Energy Chem. 46, 349 (2012).

${ }^{20}$ A. E. Dubinov and D. Yu. Kolotkov, IEEE Trans. Plasma Sci. 40, 1429 (2012).

${ }^{21}$ J. Xue, Phys. Rev. E 69, 016403 (2004).

${ }^{22}$ F. Verheest, M. A. Hellberg, and W. A. Hereman, Phys. Rev. E 86, 036402 (2012).

${ }^{23}$ X. Qi, Y. Xu, W. Duan, L. Zhang, and L. Yang, Phys. Plasmas 21, 082118 (2014).

${ }^{24}$ A. Kakad, B. Kakad, and Y. Omura, Phys. Plasmas 24, 060704 (2017).

${ }^{25}$ C. Zhou and I. H. Hutchinson, Phys. Plasmas 25, 082303 (2018).

${ }^{26}$ S. M. Hosseini Jenab and G. Brodin, Phys. Plasmas 26, 022303 (2019).

${ }^{27}$ D. Mandal and D. Sharma, Phys. Plasmas 23, 022108 (2016).

${ }^{28}$ D. N. Gao, H. Zhang, J. Zhang, Z. Z. Li, and W. S. Duan, Eur. Phys. J. D 70, 235 (2016).

${ }^{29}$ S. M. Hosseini Jenab and F. Spanier, Phys. Plasmas 24, 032305 (2017).

${ }^{30}$ F. Verheest and W. A. Hereman, J. Plasma Phys. 85, 905850106 (2019).

${ }^{31}$ C. P. Olivier, F. Verheest, and W. A. Hereman, Phys. Plasmas 25, 032309 (2018).

${ }^{32}$ A. Lotekar, A. Kakad, and B. Kakad, Phys. Plasmas 26, 100701 (2019).

${ }^{33}$ B. Xie, K. He, and Z. Huang, Phys. Plasmas 6, 3808 (1999).

${ }^{34}$ T. S. Gill, H. Kaur, and N. S. Saini, J. Plasma Phys. 71, 23 (2005).

${ }^{35}$ M. Bacha and M. Tribiche, J. Plasma Phys. 79, 569 (2013).

${ }^{36}$ C. P. Olivier, F. Verheest, and S. K. Maharaj, J. Plasma Phys. 82, 905820605 (2016).

${ }^{37}$ S. Torvén, Phys. Rev. Lett. 47, 1053 (1981).

${ }^{38}$ R. Courant, K. Friedrichs, and H. Lewy, Math. Ann. 100, 32 (1928).

${ }^{39}$ P. O. Dovner, A. I. Eriksson, R. Boström, and B. Holback, Geophys. Res. Lett. 21, 1827, https://doi.org/10.1029/94GL00886 (1994). 